\title{
Inhaled corticosteroid therapy reduces the risk of rehospitalization and all-cause mortality in elderly asthmatics
}

\author{
D.D. Sin*, J.V. Tu*,\#
}

Inhaled corticosteroid therapy reduces the risk of rehospitalization and all-cause mortality in elderly asthmatics. D.D. Sin, J.V. Tu. C) ERS Journals Ltd 2001.

ABSTRACT: Elderly patients with asthma have relatively high rates of hospitalization and mortality. Although inhaled corticosteroids have been shown to improve outcomes among younger patients with asthma, their usefulness in elderly patients has not been established. Therefore, a population-based study of patients 65 yrs of age or older, who have been hospitalized at least once with asthma in Ontario, Canada was conducted to determine the impact of inhaled corticosteroids on rehospitalization for asthma and allcause mortality rates.

Data from the Canadian Institute of Health Information was used to capture all patients 65 yrs of age and older who were hospitalized at least once, with the most responsible diagnosis of asthma in Ontario, Canada between fiscal year 1992 and 1996. This database was then linked with drug claims, physician billing and mortality databases.

In total, 6,254 consecutive elderly patients with asthma were identified. Sixty percent of these patients were given at least one prescription for inhaled corticosteroids within 90 days postdischarge from their index hospitalization for asthma. Users of inhaled corticosteroids postdischarge were $29 \%(95 \%$ confidence interval (CI) $20 \%-38 \%)$ less likely to be readmitted to hospital for asthma and $39 \%(95 \%$ CI, $20 \%-53 \%)$ less likely to experience all-cause mortality compared to those who did not receive these drugs postdischarge over a one year follow-up period.

These findings suggest that inhaled corticosteroids are beneficial in reducing the risk for rehospitalization and all-cause mortality in elderly patients with asthma who have recently been hospitalized for their disease.

Eur Respir J 2001; 17: 380-385.
*Institute for Clinical Evaluative Sciences (ICES), ${ }^{\#}$ Dept of Medicine, Sunnybrook And Women's College Health Science Center, University of Toronto, Toronto, Ontario, "Dept of Medicine, University of Alberta, Edmonton, Alberta, Canada.

Correspondence: D.D. Sin, 2E4.29 Walter C. Mackenzie Centre, University of Alberta, Edmonton, AB., Canada.

Fax: 17804076384

Keywords: Asthma hospitalization

inhaled corticosteroids mortality

Received: January 272000 Accepted after revision June 162000

This work is supported in part by ICES, which is funded by the Ontario Ministry of Health. Dr. Sin was supported by a fellowship from the Alberta Heritage Foundation for Medical Research at the time of this work. Dr. Tu is supported by a Medical Research Council of Canada Scholarship Award.
Asthma is a chronic inflammatory disorder characterized by episodic wheezing and bronchospasm. Several recent randomized studies have demonstrated the benefits of inhaled corticosteroids in reducing patient symptoms and improving lung function by suppressing airway inflammation [1, 2]. Two recent observational studies have shown that inhaled corticosteroid therapy may also reduce hospitalization rates in younger patients with asthma [3, 4]. However, the effect of inhaled corticosteroids in those 65 years of age and older has not been well established.

A recent report from the Center for Disease Control indicates that elderly patients with asthma have the highest rates of asthma mortality and hospitalization among all adult patients with this disease, highlighting the growing burden of disease in this segment of the population [5]. In 1995 alone, 55\% of all deaths and $20 \%$ of hospitalizations from asthma occurred in those 65 yrs of age or older [5]. Paradoxically, this group has been excluded from previous randomized trials of inhaled corticosteroids in asthma and extrapolation of results from the younger population to the older patients may not be appropriate for several reasons.
Older patients with asthma are more likely to have a longer duration of disease than younger patients. Since long-standing airway inflammation may lead to fixed airways obstruction, asthma of elderly patients may be less responsive to the effects of inhaled corticosteroids compared to that of younger patients [6]. Moreover, recent reports suggesting an elevated risk of significant systemic side effects from these drugs in the elderly population raise concerns about their long-term utility in the management of chronic stable asthma [7]. Using a large population-based cohort of patients, the null hypothesis that inhaled corticosteroid treatment would not be associated with a significant change in the rate of rehospitalizaton for asthma or all-cause mortality in elderly patients with asthma, was tested.

\section{Methods}

\section{Data sources}

Information from four large administrative databases in Ontario, Canada was obtained. Firstly, the 
Canadian Institute for Health Information (CIHI) hospital discharge database was searched, which contains data on all hospitalizations in Ontario, including patient demographics, admission and discharge dates, the most responsible and up to 15 secondary diagnoses as coded by the International Classifications of Diseases, ninth revision (ICD-9) [8]. Secondly, the Ontario Drug Benefit (ODB) program database was examined. The ODB program provides full dollar coverage for outpatient prescription drugs to all Ontario residents 65 yrs of age or older. All claims in this database contain a unique identification number of the medication, as well as the quantity of pills and the date dispensed. Thirdly, data on vital statistics was obtained through the Ontario Registered Person Database (RPDB), which captures the vital status of all Ontario residents. Fourthly, the Ontario Health Insurance Plan database was examined, which contains billing information on all patients who have utilized health care services in Ontario. For each claim, this database records the specialty of the billing physician, as well as the type of outpatient services that were provided.

These four databases were linked together using a common health card number present in each database, which was scrambled in order to protect patient confidentiality. The accuracy of information within these databases has been established through previous studies [9-11]. This study was approved by the local institutional review board.

\section{Study patients}

The study population was taken from residents of Ontario, Canada, 65 yrs of age or older, who between April 1, 1992 and March 31, 1997, had been hospitalized with a most responsible diagnosis of asthma. ICD-9 codes 493.0, 493.1, and 493.9 were used to identify these patients $[3,4,12]$. The index hospitalization date was defined as the patient's first admission to an acute care hospital between April 1, 1992 and March 31, 1997, with a most responsible diagnosis of asthma. The index discharge date was defined as the date on which patients were sent home from the index hospitalization. Patients who died in hospital or within 30 days of the discharge date were excluded in order to permit a reasonable window of opportunity for all patients to receive inhaled corticosteroids. Patients who were transferred to a chronic or an acute care hospital were excluded because outpatient drug information was not available for these patients.

\section{The relationships of interest}

The main association of interest was the use of inhaled corticosteroid treatment at or near the index discharge date (within 90 days of the discharge date) and the time to rehospitalization for asthma or all-cause mortality over a 12 month period. The primary outcomes of rehospitalization for asthma and allcause mortality were analysed together as well as separately. The inhaled corticosteroids relevant to this study were beclomethasone dipropionate, budesonide, fluticasone propionate, triamcinolone acetonide and flunisolide.

\section{Severity of disease and comorbidities}

Comorbidities were determined using the CIHI database. A modified Charlson Index score was calculated for each individual patient, using ICD-9 codes in the 15 secondary diagnosis field [13]. To derive this score, we took into account the presence of the following comorbid conditions in these fields: congestive heart failure (ICD-9 codes 428.x), ischemic heart disease (ICD-9 codes 410.x, 412.x), peripheral vascular disease (ICD-9 codes 443.9, 441.x, 785.4, V43.4), cerebrovascular accidents (ICD-9 codes 430.x-438.x), dementia (ICD-9 codes 290.x), rheumatological diseases (ICD-9 codes 710.0, 710.1, 710.4, 714.0-714.2, 714.81, 725.x), peptic ulcer disease (ICD-9 codes 531.x534.9), diabetes (ICD-9 codes 250.0-250.7), malignancy (ICD-9 codes 140.x-172.9, 174.x-195.8, 196.x-199.1, 200.x-208.9), renal (ICD-9 codes 582.x, 583.x, 585.x, 586.x, 588x.) and liver diseases (ICD-9 codes 571.x, 572.x) [13]. A Charlson Index score of zero denotes the absence of any comorbidities; a higher number indicates an increasing burden of comorbidities. In this study, the median Charlson score was zero with a range of $0-11$. To control for severity of pulmonary disease, the following surrogate markers were used: 1) receipt of other antiasthma medications (inhaled $\beta_{2^{-}}$ agonists and anticholinergics, oral corticosteroids and theophylline derivatives) and oral antibiotics within 90 days of the index discharge date. 2) Use of emergency room and outpatient physician services for asthma within $1 \mathrm{yr}$ prior to the index hospitalization date. Receipt of inhaled corticosteroids prior to the index hospitalization as a marker for disease severity was not used because of the potential for colinearity with the exposure variable of interest (i.e. receipt of inhaled corticosteroids postdischarge).

\section{Statistical analyses}

The means and standard deviations of continuous variables were compared using 2-tailed t-tests. Nonnormally distributed variables were compared using the Wilcoxon Rank Sum test. Ordinal and binary variables were compared using the Chi-squared test. Survival rates between patients receiving and not receiving inhaled corticosteroids were compared using the Cox proportional hazards model in order to control for differences in follow-up time and to adjust for the effects of important covariates. Because of the size of the data set, traditional statistical methods could not be relied on to aid in the selection of variables to the final model. Therefore, only those variables which were deemed a priori to be potentially important confounders of the relationship between use of inhaled corticosteroids and the outcomes of interest were used. These included age (as a continuous variable), sex, receipt of other antiasthma medications (inhaled $\beta_{2}$-agonists and anticholinergics, oral corticosteroids 
Table 1.-Characteristics of elderly patients with asthma who were and were not treated with inhaled corticosteroids following discharge

\begin{tabular}{|c|c|c|c|}
\hline & No inhaled steroids & Inhaled steroids & p-value \\
\hline Patients & 2495 (39.9) & $3759(60.1)$ & -- \\
\hline Age & $74.8 \pm 7.2$ & $73.9 \pm 6.5$ & 0.001 \\
\hline Male & $749(30.0)$ & $1244(33.1)$ & 0.007 \\
\hline Charlson Index score & $0.6 \pm 0.9$ & $0.6 \pm 0.8$ & 0.202 \\
\hline \multicolumn{4}{|c|}{$\geqslant 1$ dispensing of medications within 1 year prior to hospitalization: } \\
\hline Inhaled $\beta_{2}$-agonists & $1494(59.9)$ & $3023(80.4)$ & 0.001 \\
\hline Inhaled anticholinergics & $521(20.9)$ & $1316(35.0)$ & 0.001 \\
\hline Inhaled corticosteroids & $1101(44.1)$ & $2718(72.3)$ & 0.001 \\
\hline Oral corticosteroids & $759(30.4)$ & $1558(41.4)$ & 0.001 \\
\hline Oral theophyllines & $402(16.1)$ & $807(21.5)$ & 0.001 \\
\hline \multicolumn{4}{|c|}{ Number of physician and emergency room visits for asthma occurring within 1 year prior to index hospitalization: } \\
\hline Emergency room visit & $1.0 \pm 1.2$ & $1.5 \pm 1.3$ & 0.001 \\
\hline Outpatient physician visit & $3.9 \pm 2.2$ & $4.0 \pm 2.2$ & 0.012 \\
\hline
\end{tabular}

Data are presented as number $(\%)$ or mean $\pm \mathrm{SD}$.

and theophylline derivatives) and oral antibiotics within 90 days of the index discharge date, use of emergency room and outpatient physician services for asthma within $1 \mathrm{yr}$ prior to the index hospitalization date, and the Charlson comorbidity score. There were missing data on outpatient physician and emergency department visits on $5 \%$ of patients. Analyses were conducted with and without these patients. Because the findings were similar, data are presented on patients who did not have any missing data. Graphing techniques (i.e. comparison of estimated $\log -\log (-\ln (-\ln ))$ survival curves) were used to check for the proportional hazards assumption [14] and it was adequately met.

\section{Results}

In total, 6,254 elderly patients with asthma meeting the entry criteria were identified; $3,759(60 \%)$ of these patients filled at least one prescription for an inhaled corticosteroid in the first 90 days following the index discharge date. The characteristics of the asthma study population are listed in table 1 stratified according to whether or not they received at least one dispensing for inhaled corticosteroids within the first 90 days postdischarge. Those treated with inhaled corticosteroids postdischarge had a significantly greater number of physician and emergency room visits for asthma within $1 \mathrm{yr}$ prior to their index hospitalization. Moreover, they had greater utilization of other antiasthma medications before and after discharge from their index hospitalization (tables 1 and 2). Since use of antiasthma medica- tions is a marker for disease severity, those treated with inhaled corticosteroids postdischarge were likely to have greater asthma burden than those not treated with inhaled corticosteroids [15].

In an unadjusted analysis, the use of inhaled corticosteroids was associated with an $8 \%(95 \% \mathrm{CI}$, $-2 \%-17 \%$ ) relative reduction in the combined rate of recurrent hospitalization for asthma or all-cause mortality, compared to those who did not use these medications. When age, sex, comorbidity score, and use of other antiasthma medications and physician services were controlled for, use of inhaled corticosteroids was associated with a $32 \%(95 \% \mathrm{CI}, 23 \%-39 \%)$ relative rate reduction for recurrent hospitalization or all-cause mortality (fig. 1). In contrast, receipt of all other antiasthma medications was associated with a neutral or increased relative risk for rehospitalization or mortality.

The association between the use of inhaled corticosteroids and all-cause mortality was stronger than that between inhaled corticosteroids and rehospitalization. In an adjusted analysis, the use of inhaled corticosteroids postdischarge was associated with a 39\% (95\% CI, 20\%-53\%) reduction in the mortality rate, compared to those who did not receive inhaled corticosteroids. In contrast, a $29 \%(95 \%$ CI, $20 \%-$ $38 \%$ ) relative reduction in the rate for rehospitalization for asthma was observed.

In patients who received at least one prescription for inhaled corticosteroids within $1 \mathrm{yr}$ prior to the index hospitalization, the use of inhaled cortiocosteroids 90 days postdischarge was associated with $41 \%$ (95\% CI,

Table 2.-Receipt of at least 1 prescription of anti-asthma medications within 90 days of discharge from index hospitalization in those treated and not treated with inhaled corticosteroids postdischarge

\begin{tabular}{lcrr}
\hline & No inhaled steroids & Inhaled steroids & p-value \\
\hline Patients & $2495(39.9)$ & $3759(60.1)$ & - \\
Inhaled $\beta_{2}$-agonists & $933(37.4)$ & $3229(85.9)$ & 0.001 \\
Inhaled anticholinergics & $483(19.4)$ & $1929(51.3)$ & 0.001 \\
Oral corticosteroids & $619(24.8)$ & $1631(43.4)$ & 0.001 \\
Oral theophyllines & $254(10.2)$ & $691(18.4)$ & 0.001 \\
Antibiotics & $786(31.5)$ & $1712(45.5)$ & 0.001 \\
\hline
\end{tabular}

Data are presented as number (\%). 


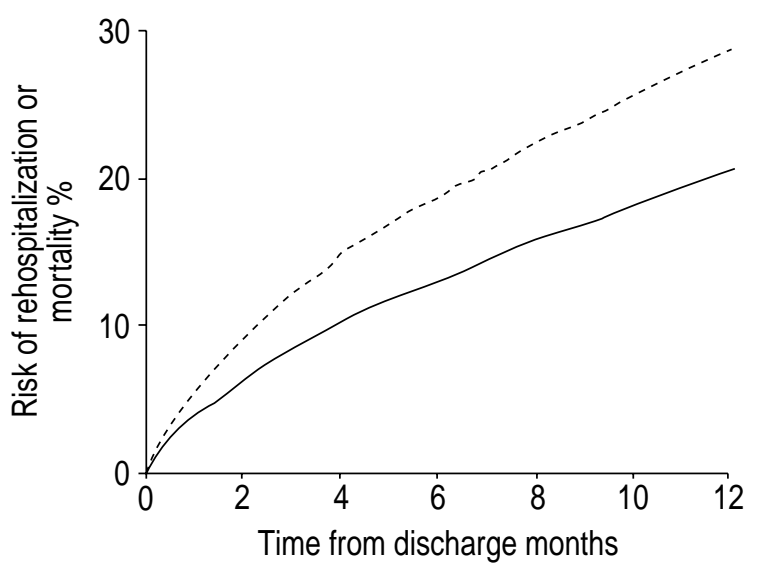

Fig. 1. - Adjusted probability of recurrent hospitalization or allcause mortality among elderly patients with asthma who received (-) or did not receive (---) inhaled corticosteroids postdischarge. In the adjusted model, age (as a continuous variable), sex, receipt of other antiasthma medications (inhaled $\beta_{2}$-agonists and anticholinergics, oral corticosteroids and theophylline derivatives) and oral antibiotics within 90 days of the index discharge date, use of emergency room and outpatient physician services for asthma within one year prior to the index hospitalization date, and Charlson comorbidity score were all controlled for. There was a statistically significant difference $(p<0.001)$ in the slopes of the two survival curves.

$32 \%-49 \%$ ) fewer recurrent hospitalizations for asthma or deaths, compared to nonuse of these medications. In elderly asthmatic patients, who did not receive any inhaled corticosteroids within $1 \mathrm{yr}$ prior to the index hospitalization, the use of inhaled corticosteroids postdischarge was associated with a $21 \%(95 \% \mathrm{CI}$, $3 \%-35 \%$ ) relative reduction in the rate of asthma hospitalizations or all-cause mortality, compared to those who did not use these medications.

In patients without any comorbidites (i.e. a Charlson score of zero), use of inhaled corticosteroids postdischarge was associated with a 39\% (95\% CI, 29\%$48 \%$ ) relative risk reduction in hospitalizations for asthma or all-cause mortality, compared to those who did not use these medications. In patients with a Charlson score of $\geqslant 1$, the use of inhaled corticosteroids was associated with a $24 \%(95 \%$ CI, 10-36\%) risk reduction for recurrent hospitalization or all-cause mortality, compared to nonuse.

\section{Discussion}

The most important finding in this study was that inhaled corticosteroid treatment postdischarge was associated with a $32 \%$ reduction in the combined rate of rehospitalization or all-cause mortality, compared to nonusers, in a group of elderly patients with asthma. This result is consistent with the known benefits of inhaled corticosteroids on lung function, symptom scores and health-related quality of life $[1,2]$. Moreover, these results are in keeping with those of Donahue et al. [3] and Blais et al. [4] who demonstrated the benefits of inhaled corticosteroids in preventing hospitalization in younger patients with asthma. Their findings have been extended by showing that inhaled corticosteroids are also effective in reducing hospitalization as well as all-cause mortality in asthma patients $65 \mathrm{yrs}$ of age and older. The present findings are important because previous randomized studies have systematically excluded elderly patients with asthma. The effect of inhaled anti-inflammatory medications in the elderly population, therefore, was not previously known.

The magnitude of the effect on hospitalization and mortality rate was slightly smaller than what has been previously reported for younger patients with asthma $[3,4,16]$. This may be related to the fact that many elderly patients have a long-standing history of asthma, which may result in irreversible airflow obstruction [6]. Moreover, the elderly population has a higher prevalence of other chronic lung conditions such as emphysema and bronchiectasis. Coexistence of these lung diseases may diminish the effectiveness of inhaled corticosteroids because these conditions are generally refractory to the effects of inhaled corticosteroids. Asthma may also be mistaken for COPD in the elderly population because clinical presentations may be quite similar [17]. Since recent studies of inhaled corticosteroids in the COPD population suggest these drugs may not be effective in this condition [18, 19], diagnostic misclassification may have also contributed to the lower relative risk reduction from inhaled corticosteroids in our cohort of patients.

Although inhaled corticosteroid therapy was associated with a reduction in both rehospitalization or allcause mortality in our study, their effect on mortality was greater than that for rehospitalization. These observations are consistent with what has been previously published for younger asthmatics. In a cohort study from Saskatchewan, BLAIs et al. [4] reported a $40 \%$ relative risk reduction for rehospitalization for asthma in patients treated with inhaled corticosteroids compared to those not treated with inhaled corticosteroids. However, for the same cohort of patients, there was a striking $90 \%$ relative risk reduction for asthma mortality, suggesting that inhaled steroids are more powerful in preventing mortality than reducing the risk for rehospitalization [16]. The differential impact of these efficacious therapies on mortality and hospitalization rates is probably explainable on the basis of disease severity; those with the greatest disease severity are likely to derive the largest benefits from these medications.

Another important observation was that only $60 \%$ of elderly patients with asthma received at least one dispensing of inhaled corticosteroids postdischarge. Previous studies have also demonstrated that inhaled corticosteroids may be underused in the community [20, 21]. The present observations, however, are unique in that this study was conducted in a health care system that provided free medications to all members of the cohort during the study period. This suggests that a suboptimal rate of utilization of inhaled corticosteroids occurs even in settings where there are minimal financial barriers to medication access. A paucity of data demonstrating the benefits of inhaled corticosteroids in the elderly population and a growing concern over the side effect profile of these medications in the elderly may be contributing to a suboptimal rate 
of utilization of inhaled corticosteroids in this population of patients [8].

A major limitation of this and other observational studies is the uncertainty over whether one can completely adjust for confounding factors. For example, were patients given inhaled steroids more likely to be asthmatics while those not given these medications more likely to have COPD? A previous study in Ontario, Canada of previously hospitalized elderly patients showed that the rate of use of inhaled corticosteroids is very similar between patients with asthma and COPD [22]. Thus, even if there were significant diagnostic misclassification, it would have been nondifferential in nature, leading to an underestimation of the point estimates. This type of misclassification, however, would not change the overall conclusion of this study. If some patients did not receive inhaled corticosteroids postdischarge because they had already filled in a prescription for inhaled corticosteroid before their index hospitalization, this would be another potential source of "exposure" misclassification. It was reassuring, however, that the inverse association between use of inhaled corticosteroids and hospital-free survival was also present in elderly asthmatic patients who had not received any inhaled corticosteroids within $1 \mathrm{yr}$ prior to their index hospitalization, suggesting that exposure misclassification was unlikely to explain the present findings. Another limitation of this study was that no information on the cause of death was available. Because patients who were recently hospitalized for an asthma exacerbation were studied, it is likely that asthma was a major cause of death for decedents in our cohort. However, since inhaled corticosteroids are unlikely to impact on deaths from unrelated causes such as ischemic heart disease, it is possible that by considering only all-cause mortality, the effect of these medications in reducing asthma-specific mortality may have been underestimated.

With asthma, there is no universally accepted method for controlling disease severity. Although pulmonary function tests measured during clinical stability may be of some use in predicting outcomes, assessment of lung functions during exacerbations do not have good predictive value for asthma severity or outcomes $[23,24]$. On the other hand, health services information such as use of airway medications and prior physician/ emergency visits, which indirectly capture severity of disease, correlate well with morbidity and mortality in asthma [25-27]. Indeed, in one study, the best measure of severity was prior history of hospitalization or emergency department visits, and oral systemic steroid use [28]. In another study, the strongest markers for death or readmission were a history of hospitalization and use of antiasthma medications, including inhaled as well as oral steroids in the previous 12 months [28]. These results have been replicated in other studies [29, 30]. In the present analysis, all of these prognostic factors have been controlled for. Indeed, those who received inhaled corticosteroids had more prior physician visits, including emergency room admissions, and had greater utilization of other antiasthma medications, suggesting that as a group, these patients had a more severe form of asthma than those who did not receive these drugs. Therefore, while the absence of pulmonary function results is a limitation of this study, the database contained other important variables, which allowed for us to reasonably control for disease severity in the patients.

Morbidity and mortality related to asthma have increased over the last decade in developing countries $[31,32]$ and represent an important source of resource allocation [33]. The present study suggests that inhaled corticosteroid therapy can improve survival and reduce hospitalization in elderly patients with asthma who have previously been hospitalized by their disease. These results highlight the need to increase the rate of use of inhaled corticosteroids in elderly patients with moderate to severe asthma, which is in keeping with the recommendations of the National Educational Program on Asthma [34]. The fact that only $60 \%$ of elderly patients in the study received inhaled corticosteroids postdischarge, underscores the need for such programs to enhance their utilization in the community.

\section{References}

1. Haahtela T, Jarvinen M, Kava T, et al. Comparison of a beta-2 agonist, terbutaline, with an inhaled corticosteroid, budesonide, in newly detected asthma. $N$ Engl J Med 1991; 325: 388-392.

2. Haahtela T, Jarvinen M, Kava T, et al. Effects of Reducing or Discontinuing Inhaled Budesonide in Patients with Mild Asthma. N Engl J Med 1994; 331: $700-705$.

3. Donahue JG, Weiss ST, Livingston JM, Goetsch MA, Greinder DK, Platt R. Inhaled steroids and the risk of hospitalization for asthma. JAMA 1997; 277: $887-$ 891.

4. Blais L, Ernst P, Boivin JF, Suissa S. Inhaled corticosteroids and the prevention of readmission to hospital for asthma. Am J Respir Crit Care Med 1998; 158: $126-132$.

5. Mannino DM, Homa DM, Pertowski CA. Mor Mortal Wkly Rep CDC Surveill Summ 1998; 47: 1-27.

6. Braman SS, Kaemmerlen JT, Davis SM. Asthma in the elderly. A comparison between patients with recently acquired and long standing disease. Am Rev Respir Dis 1991; 143: 336-340.

7. Garbe E, Suissa S, LeLorier J. Association of inhaled corticosteroid use with cataract extraction in elderly patients. JAMA 1998; 280: 539-543.

8. Department of Health and Human Services. 1980. The international classification of diseases, 9th revision, clinical modification: ICD-9-CM. Vol. 1. Diseases: alphabetic index. Washington, D.C.

9. Chan B, Anderson GM, Theriault ME. Fee code creep among general practitioners and family physicians in Ontario: why does the ratio of intermediate to minor assessments keep climbing? CMAJ 1998; 158: 749 754.

10. Jaglal SB, Tu JV, Naylor CD. Higher in-hospital mortality in female patients following coronary artery bypass surgery: a population based study. Provincial Adult Cardiac Care Network of Ontario. Clin Invest Med 1995; 18: 99-107.

11. Tu JV, Hannan EL, Anderson GM, et al. The fall and rise of carotid endarterectomy in the United States and Canada. N Engl J Med 1998; 339: 1441-1447. 
12. Cydulka RK, McFadden ER, Emerman CL, Sivinski LD, Pisanelli W, Rimm AA. Patterns of hospitalization in elderly patients with asthma and chronic obstructive pulmonary disease. Am J Respir Crit Care Med 1997; 156: $1807-1812$.

13. Deyo RA, Cherkin DC, Ciol MA. Adapting a clinical comorbidity index for use with ICD-9-CM administrative databases. J Clin Epidemiol 1992; 45: $613-629$.

14. Kleinbaum DG. Survival analysis: a self-learning text. New York, Springer-Verlag, 1996; 133-139.

15. Grana J, Preston S, McDermott PD, Hanchak NA. The use of administrative data to riskstratify asthmatic patients. Am J Med Qual 1997; 12: $113-119$.

16. Ernst P, Spitzer WO, Suissa S. Risk of fatal and nearfatal asthma in relation to inhaled corticosteroid use. JAMA 1992; 268: 3462-3464.

17. Fanta CH. Asthma in the elderly. J Asthma 1989; 26: 87-97.

18. Vestbo J, Sorensen T, Lange P, Brix A, Torre P, Viskum K. Long-term effect of inhaled budesonide in mild and moderate chronic obstructive pulmonary disease: a randomised controlled trial. Lancet 1999; 353: $1819-1823$.

19. Pauwels RA, Lofdahl CG, Laitinen LA. Long-term treatment with inhaled budesonide in persons with mild chronic obstructive pulmonary disease who continue smoking. European Respiratory Society Study on Chronic Obstructive Pulmonary Disease. $N$ Engl J Med 1999; 340: 1948 - 1953.

20. Joyce DP, McIvor RA. Use of inhaled medications and urgent care services. Study of Canadian asthma patients. Can Fam Physician 1999; 45: 1707-1713.

21. Gottlieb DJ, Beiser AS, O'Connor GT. Poverty, race, and medication use are correlates of asthma hospitalization rates. A small area analysis in Boston. Chest 1995; 108: 28-35.

22. Jackevicius C, Joyce DP, Kesten S, Chapman KR. Prehospitalization inhaled corticosteroid use in patients with COPD or asthma. Chest 1997; 111: 296-302.

23. Fuso L, Incalzi RA, Pistelli R, et al. Predicting mortality of patients hospitalized for acutely exacerbated chronic obstructive pulmonary disease. Am J Med 1995; 98: 272-277.

24. Kucera CM, Greenberger PA, Yarnold PR, Choy AC, Levenson T. An attempted prospective testing of an asthma severity index and a quality of life survey for 1 year in ambulatory patients with asthma. Allergy Asthma Proc 1999; 20: 29-38.

25. Wasilewski Y, Clark NM, Evans D, Levison MJ, Levin B, Mellins RB. Factors associated with emergency department visits by children with asthma: implications for health education. $\mathrm{Am}$ J Public Health 1996; 86: 1410-1415.

26. LeSon S, Gershwin ME. Risk factors for asthmatic patients requiring intubation. III. Observations in young adults. J Asthma 1996; 33: 27-35.

27. Li D, German D, Lulla S, Thomas RG, Wilson SR. Prospective study of hospitalization for asthma. A preliminary risk factor model. Am J Respir Crit Care Med 1995; 151: 647-655.

28. Crane J, Pearce N, Burgess C, Woodman K, Robson B, Beasley R. Markers of risk of asthma death or readmission in the 12 months following a hospital admission for asthma. Int $J$ Epidemiol 1992; 21: 737 744.

29. Meir CR, Jick H. Drug use and pulmonary death rates in increasingly symptomatic asthma patients in UK. Thorax 1997; 52: 612-617.

30. Guite HF, Dundas R, Burney PG. Risk factors for death and asthma, chronic obstructive pulmonary disease, and cardiovascular disease after a hospital admission for asthma. Thorax 1999; 54: $301-307$.

31. Erzen D, Roos LL, Manfreda J, Anthonisen NR. Changes in asthma severity in Manitoba. Chest 1995; 108: $16-23$.

32. Sears MR. Descriptive epidemiology of asthma. Lancet 1997; 350: SII 1-4.

33. Weiss KB, Gergen PJ, Hodgson TA. An economic evaluation of asthma in the United States. $N$ Engl $J$ Med 1992; 326: $862-866$.

34. U.S. Department of Health and Human Services. National Asthma Education and Prevention Program. The Expert Panel Report 2: Guidelines for the Diagnosis and Management of Asthma. 1997. NIH Publication No. 97-4051, 1-153. 